Y. Komori

Nagoya Math. J.

Vol. 77 (1980), 33-39

\title{
COMPLETENESS OF TWO THEORIES ON ORDERED ABELIAN GROUPS AND EMBEDDING RELATIONS
}

\author{
YUICHI KOMORI
}

\section{§ 0. Introduction}

The first order language $\mathscr{L}$ that we consider has two nullary function symbols 0,1 , a unary function symbol - a binary function symbol +, a unary relation symbol $0<$, and the binary relation symbol $=$ (equality). Let $\mathscr{L}^{\prime}$ be the language obtained from $\mathscr{L}$, by adding, for each integer $n>0$, the unary relation symbol $n \mid$ (read " $n$ divides"). The terms $1+$ $\cdots+1$ and $t+\cdots+t$ ( 1 and $t$ repeated $n$ times) will be written as $n$ and $n t$, the term $t+(-s)$ as $t-s$, the atomic formula $0<t-s$ as $t<s$, and the formulas $u<t \wedge t<s$ and $t=s \vee t<s$ as $u<t<s$ and $t \leqq s$, respectively. We now give some axiom systems for abelian groups with a semidiscrete total ordering.

(a) The axioms for abelian groups:

$$
\begin{aligned}
& (x+y)+z=x+(y+z) \\
& x+y=y+x \\
& x+0=x \\
& x-x=0 .
\end{aligned}
$$

(b) The axioms for a total ordering compatible with group structures:

$$
\begin{aligned}
& 0<x \wedge 0<y \rightarrow 0<x+y \\
& \neg(0<x \wedge 0<-x) \\
& x=0 \vee 0<x \vee 0<-x .
\end{aligned}
$$

(c) The axioms for a semi-discrete ordering:

$$
\begin{aligned}
& 0<1 \\
& 2 x<1 \vee 1<2 x
\end{aligned}
$$

(d) The axioms for infinitesimals:

$$
2 x<1 \rightarrow n x<1 \text { for each } n>2 \text {. }
$$

The axioms for $n \mid$ are

Received October 26, 1978. 
(e) $n \mid x \leftrightarrow \exists y \exists z(-1<2 z<1 \wedge x=n y+z)$ for each $n>0$, and

(f) $n|x \vee n| x+1 \vee n|x+2 \vee \cdots \vee n| x+n-1$ for each $n>1$.

(g) The axioms for divisable infinitesimals:

$-1<2 x<1 \rightarrow \exists y(x=n y)$ for each $n>1$.

(h) The axiom for discrete orderings:

$\neg(0<x<1)$.

(i) The axiom for existence of infinitesimals:

$\exists x(0<x<1)$.

The language of the theory $S$ is $\mathscr{L}$. The set of axioms of $S$ is (a) $\cup($ b) $\cup(c) \cup(d)$ (We will write (a, b, c, d) for this set in future.). The language of the theory $\boldsymbol{D}$ is $\mathscr{L}$ as well. The set of axioms of $\boldsymbol{D}$ is $\boldsymbol{S} \cup(\mathrm{h})$ (equivalent to $(\mathrm{a}, \mathrm{b}, \mathrm{c}, \mathrm{h})$ ), which is equivalent to $(\mathrm{a}, \mathrm{b}) \cup\{0<x \leftrightarrow x=$ $1 \vee 1<x\}$. We call a model of $S$ (or $D$ ) an abelian group with a semidiscrete (or discrete) total ordering. The languages of the theories $S \boldsymbol{S}, \boldsymbol{S C}$ and $D C$ are all $\mathscr{L}^{\prime}$. The sets of axioms of $S S, S C$ and $D C$ are $S \cup(\mathrm{e}, \mathrm{f})$, $S S \cup(\mathrm{g}, \mathrm{i})$ and $S S \cup(\mathrm{g}, \mathrm{h})$ (equivalent to $D \cup(\mathrm{e}, \mathrm{f})$ ), respectively.

Let $Z$ and $Q$ be the set of integers and the set of rationals, respectively. Consider the group $Z Q=Z \times Q$ ordered as follows: $0<(x, y)$ if and only if either $0<x$ or $x=0$ and $0<y . \quad Z Q$ is a model of $S$ which contains $Z$ as a submodel (identifying $(n, 0)$ with $n$ ). Of course, $Z$ is a model of $D$, but $Z Q$ is not a model of $D$. It is clear that each model of $\boldsymbol{S}$ is also a model of (e), or, more precisely, given a model of $\boldsymbol{S}$, there is a unique value of $n \mid$ so that (e) is satisfied. $Z Q$ is a model of $S C$ as well.

It is known that $D C$ allows elimination of quantifiers and that it is complete (cf. Kreisel and Krivine [2] p. 54. There exists an error in the proof, but it is easy to correct the error.). In $\S 1$, we shall show that $S C$ allows elimination of quantifiers and that it is complete and decidable.

In $\S 2$, we shall show that any model of $S(S S, D)$ can be embedded in some model of $S S$ (SC, DC, respectively). Embedding relations will be used to show some results on the first order semantics for $\mathscr{L}$ and $\mathscr{L}^{\prime}$. One of them is that for any universal formula $F$ of $\mathscr{L}^{\prime}$ and any model $A$ of $S S \cup(\mathrm{i}), F$ is valid in $Z Q$ if and only if $F$ is valid in $A$. In the paper [1], we shall make use of it for giving a complete description of superŁukasiewicz propositional logics.

$Z$ is a model of $S \boldsymbol{S} \cup(\mathrm{g})$, but not a model of $\boldsymbol{S C}$. Therefore, (i) is not a consequence of the set $(\mathrm{a}, \mathrm{b}, \mathrm{c}, \mathrm{d}, \mathrm{e}, \mathrm{f}, \mathrm{g})$. Consider the group $Z Z=$ $Z \times Z$ ordered as follows: $0<(x, y)$ if and only if either $0<x$ or $x=0$ 
and $0<y$. This group (identifying $(n, 0)$ with $n$ ) is a model of $\boldsymbol{S S} \cup(\mathrm{i})$, but not a model of $S C$. Hence, (g) is not a consequence of the set (a, b, c, d, e, f, i).

\section{$\S 1$. Elimination of quantifiers}

To show that $S C$ admits elimination of quantifiers, we consider a formula $F$ of the form $\exists x\left(\alpha_{1} \wedge \cdots \wedge \alpha_{n}\right)$ where each $\alpha_{i}$ is an atomic formula of $\mathscr{L}^{\prime}$ or the negation of an atomic formula of $\mathscr{L}^{\prime}$. Thus $\alpha_{i}$ is of one of the forms $t=s, t \neq s, 0<t, \neg(0<t), n \mid t$ or $\neg(n \mid t)$.

In this section, the derivations from $\boldsymbol{S S}$ are done without notice. As we shall prove by the way that $D C$ admits elimination of quantifiers, we notice the use when we use (g) or (i). $t=s, t \neq s, \neg(0<t)$ and $\neg(n \mid t)$ are equivalent to $t-s=0,0<t-s \vee 0<s-t, t=0 \vee 0<-t$ and $n|t+1 \vee \cdots \vee n| t+n-1$, respectively. Hence we can suppose that each $\alpha_{i}$ is of one of the forms $t=0,0<t$ or $n \mid t$.

Each term $t$ can be written in the form $p x+s$ with $p \in Z$ and $s$ a term which does not contain $x$. If $p=0$, the atomic formula can be taken out of the scope of $\exists x$.

Thus the formula $F$ can be written in the form

$$
\begin{aligned}
& \exists x\left(p_{1} x<t_{1} \wedge \cdots \wedge p_{j} x<t_{j} \wedge u_{1}<q_{1} x \wedge \cdots \wedge u_{k}<q_{k} x \wedge\right. \\
& \left.\quad r_{1} x=v_{1} \wedge \cdots \wedge r_{l} x=v_{l} \wedge n_{1}\left|s_{1} x-w_{1} \wedge \cdots \wedge n_{m}\right| s_{m} x-w_{m}\right)
\end{aligned}
$$

where the $p, q, r, s$ are in $N$ (the set of natural numbers which does not contain 0 ) and $t, u, v, w$ are terms which do not contain $x$.

For any $k \in N, t=u, t<u$ and $n \mid t$ are equivalent to $k t=k u, k t<k u$ and $k n \mid k t$, respectively. Hence, taking the least common multiple (l.c.m.) of $p_{1}, \cdots, p_{j}, q_{1}, \cdots, q_{k}, r_{1}, \cdots, r_{l}, s_{1}, \cdots, s_{m}$ we can suppose that $p_{1}=\cdots$ $=p_{j}=q_{1}=\cdots=q_{k}=r_{1}=\cdots=r_{l}=s_{1}=\cdots=s_{m}=p$ in the formula $(*)$. Then the formula $(*)$ is equivalent to

$$
\begin{aligned}
& \exists x\left(x<t_{1} \wedge \cdots \wedge x<t_{j} \wedge u_{1}<x \wedge \cdots \wedge u_{k}<x \wedge\right. \\
& \left.\quad x=v_{1} \wedge \cdots \wedge x=v_{l} \wedge n_{1}\left|x-w_{1} \wedge \cdots \wedge n_{m}\right| x-w_{m} \wedge \exists y(x=p y)\right) .
\end{aligned}
$$

It follows from $S S \cup(\mathrm{g})$ that $\exists y(x=p y)$ is equivalent to $p \mid x . \quad$ If $l \geqq 1$, then the above formula equivalent to

$$
\begin{aligned}
v_{1}<t_{1} & \wedge \cdots \wedge v_{1}<t_{j} \wedge u_{1}<v_{1} \wedge \cdots \wedge u_{k}<v_{1} \wedge \\
v_{1} & =v_{2} \wedge \cdots \wedge v_{1}=v_{l} \wedge n_{1}\left|v_{1}-w_{1} \wedge \cdots \wedge n_{m}\right| v_{1}-w_{m} \wedge p \mid v_{1}
\end{aligned}
$$


which is quantifier free. Hence we can assume that $l=0$. If $j \geqq 2$, the formula $F$ is equivalent to

$$
\left(t_{1}<t_{2} \wedge \exists x\left(x<t_{1} \wedge x<t_{3} \wedge \cdots\right)\right) \vee\left(t_{2}<t_{1} \wedge \exists x\left(x<t_{2} \wedge x<t_{3} \wedge \cdots\right)\right)
$$

and we are reduced to the case of a formula with $j-1$ atomic formulas of the form $x<t$. Hence we can assume that $j \leqq 1$. Similarly, we can assume that $k \leqq 1$.

Suppose that $j=k=1$. Let $n$ be 1.c.m. of $n_{1}, n_{2}, \cdots, n_{m}, p$. Let $C_{q}$ be the formula

$$
\begin{gathered}
\exists y \exists z\left(0<n y+z+q<t_{1}-u_{1} \wedge-1<2 z<1\right) \wedge n_{1} \mid q+u_{1}-w_{1} \wedge \\
\cdots \wedge n_{m}\left|q+u_{1}-w_{m} \wedge p\right| q+u_{1} \quad(q=0,1,2, \cdots, n-1) .
\end{gathered}
$$

Then, $F$ is equivalent to $C_{0} \vee C_{1} \vee \cdots \vee C_{n-1}$.

It suffices to show that $\exists y \exists z(0<n y+z+q<t \wedge-1<2 z<1)$ is equivalent to some quantifier free formula. It follows from $S S \cup(\mathrm{g})$ that it is equivalent to $\exists y(0<n y+q<t)$. If $q=0$, then it is equivalent to $0<t$ in $S C$ (equivalent to $n<t$ in $D C$ ). If $0<q \leqq n-1$, then it is equivalent to $-1<2(t-q)$ in $S C$ (equivalent to $q<t$ in $D C$ ).

When $j=0$ or $k=0, F$ is equivalent to $E_{0} \vee E_{1} \vee \cdots \vee E_{n-1}$ where $E_{q}$ is the formula $n_{1}\left|q+u_{1}-w_{1} \wedge \cdots \wedge n_{m}\right| q+u_{1}-w_{m} \wedge p \mid q+u_{1}$.

This completes our proof that $S C$ (and $D C$ ) allows elimination of quantifiers.

Because any atomic formula without variables is equivalent to $0=0$ or $0 \neq 0$, any quantifier free formula without variables is equivalent to $0=0$ or $0 \neq 0$. Hence any closed formula is equivalent to $0=0$ or $0 \neq 0$. Therefore $S C$ (and $D C$ ) is complete.

Theorem 1.1. Both theories SC and DC allow elimination of quantifiers, and they are complete and decidable.

\section{§2. Embedding relations}

TheOREm 2.1. Any model of SS can be embedded in some model of $\mathbf{S C}$.

Proof. Let $A$ be a model of $S S$. When $A$ satisfies (h), we consider $A \times Q$ ordered as $0<(x, y)$ if and only if either $0<x$ or $x=0$ and $0<y$. Then $A \times Q$ is a model of $S C$ and the mapping $f: A \rightarrow A \times Q$ such that $f(x)=(x, 0)$ is an embedding of $A$ in $A \times Q$. Suppose $A$ does not satisfy (h), that is, satisfies (i). Let $B$ be the set $\{(x, n)|n| x$ and $n>0$ and $x \in A\}$. 
We define functions,-+ and a relation $0<$ on $B$ as follows: $-(x, n)$ $=(-x, n),(x, m)+(y, n)=(n x+m y, m n), 0<(x, n)$ if and only if $0<x$. The relation $\sim$ on $B$ defined by $(x, m) \sim(y, n)$ if and only if $n x=m y$ is a congruence relation. $B / \sim$ is a model of $S C$ and the mapping $g: A \rightarrow B / \sim$ such that $g(x)=[(x, 1)]$ (equivalence class containing $(x, 1)$ ) is an embedding of $A$ in $B / \sim$.

Q.E.D.

By the Embedding Theorem (cf. [2] p. 40) and the fact that $Z Q$ is a model of complete theory $S C$, we have

Corollary 2.2. For any universal formula $F$ of $\mathscr{L}^{\prime}, F$ is valid in $Z Q$ if and only if $F$ is valid in every model of $\boldsymbol{S S}$.

The following corollary is used for giving a complete description of super-Łukasiewicz propositional logics in a subsequent paper [1].

Corollary 2.3. For any universal formula $F$ of $\mathscr{L}^{\prime}$ and any model $A$ of $S S \cup(i), F$ is valid in $Z Q$ if and only if $F$ is valid in $A$.

Proof. By Corollary 2.2, $F$ is valid in $A$ if $F$ is valid in $Z Q$. Conversely, suppose that $F$ is valid in $A . Z Z$ can be embedded in any model of $S S \cup(i)$. Hence $F$ is valid in $Z Z$. Any finitely generated submodel of $Z Q$ is isomorphic to $Z$ or $Z Z$. Hence it can be embedded in $Z Z$. Therefore, $F$ is valid in $Z Q$.

Q.E.D.

LEMMA 2.4. For any model $A$ of $\boldsymbol{S}$ and any elements $x_{1}, x_{2}, \cdots, x_{q}$ of $A$, if $m+n_{1} x_{1}+\cdots+n_{q} x_{q}=0$, then $m=n_{1}=\cdots=n_{q}=0$ or there exist elements $y_{1}, y_{2}, \cdots, y_{q-1}$ of $A$ and integers $k_{i j}(1 \leqq i \leqq q, 0 \leqq j \leqq q-1)$ such that $x_{i}=k_{i 0}+\sum_{j=1}^{q-1} k_{i j} y_{j}$ for any $i(1 \leqq i \leqq q)$.

Proof. Choosing the signs of $x_{1}, x_{2}, \cdots, x_{q}$ suitably, we can assume that $n_{1}, n_{2}, \cdots, n_{q}$ are positive or zero. We prove this lemma by induction on $n_{1}+n_{2}+\cdots+n_{q}$.

Case 1. Suppose that at least two of $n_{1}, n_{2}, \cdots, n_{q}$ are positive. We can assume that $0<n_{1} \leqq n_{2}$. Then we have

$$
m+n_{1}\left(x_{1}+x_{2}\right)+\left(n_{2}-n_{1}\right) x_{2}+n_{3} x_{3}+\cdots+n_{q} x_{q}=0 .
$$

By the hypothesis of induction, there exist elements $y_{1}, \cdots, y_{q-1}$ of $A$ and integers $p_{i j}$ such that 


$$
x_{1}+x_{2}=p_{10}+\sum_{j=1}^{q-1} p_{1 j} y_{j} \quad \text { and } \quad x_{i}=p_{i 0}+\sum_{j=1}^{q-1} p_{i j} y_{j} \quad(i=2,3, \cdots, q) .
$$

Then we have $x_{1}=\left(p_{10}-p_{20}\right)+\sum_{k=1}^{q-1}\left(p_{1 j}-p_{2 j}\right) y_{j}$. It completes our proof of Case 1 that we put $k_{1 j}=p_{1 j}-p_{2 j}(0 \leqq j \leqq q-1)$ and $k_{i j}=p_{i j}(2 \leqq i$ $\leqq q, 0 \leqq j \leqq q-1)$.

Case 2. We can assume that $n_{1} \neq 0$ and $n_{2}=n_{3}=\cdots=n_{q}=0$. Since it follows from $S$ that $\forall x(m x \neq n)$ for every mutually prime integers $m, n$, $n_{1}$ is a factor of $m$. Hence, we have $x_{1}=-m / n_{1}$. Put $y_{i}=x_{i+1}(i=1$, $2, \cdots, q-1)$.

Q.E.D.

TheOREM 2.5. Any model of $\boldsymbol{S}$ can be embedded in some model of $\boldsymbol{S S}$.

Proof. It suffices to show that any model of $S$ generated by a finite set can be embedded in some model of $\boldsymbol{S S}$ (cf. Theorem 13 in [1] p. 41). Let $A$ be a model of $S$ which have $n$ generators $a_{1}, a_{2}, \cdots, a_{n}$ but can not be generated by $n-1$ generators. We define a relation $0<$ on $Z \times Q^{n}$ as follows: $0<\left(m, q_{1}, \cdots, q_{n}\right)$ if and only if $0<p m+\left(p q_{1}\right) a_{1}+\cdots+\left(p q_{n}\right) a_{n}$ where $p$ is 1.c.m. of denominators of $q_{1}, \cdots q_{n}$,

In order to prove that $Z \times Q^{n}$ is a model of $S S$, it suffices to show that $m=k_{1}=\cdots=k_{n}=0$ if $m+k_{1} a_{1}+\cdots+k_{n} a_{n}=0$. By Lemma 2.4, $a_{1}, a_{2}, \cdots, a_{n}$ can be generated by $n-1$ generators if $m+k_{1} a_{1}+\cdots+$ $k_{n} a_{n}=0$, and $m \neq 0$ or $k_{i} \neq 0$ for some $i$. Hence, $Z \times Q^{n}$ is a model of SS.

Let $f$ be a function from $A$ into $Z \times Q^{n}$ such that $f\left(m+k_{1} a_{1}+\cdots\right.$ $\left.+k_{n} a_{n}\right)=\left(m, k_{1}, \cdots, k_{n}\right)$. Then $f$ is an embedding of $A$ in $Z \times Q^{n}$. Q.E.D.

By Theorem 2.1 and Theorem 2.5, we have

TheOREm 2.6. Any model of $\boldsymbol{S}$ can be embedded in some model of $\boldsymbol{S C}$.

The following corollary can be proved similarly to Corollary 2.3.

Corollary 2.7. For any universal formula $F$ of $\mathscr{L}$ and any model $A$ of $S \cup(\mathrm{i}), F$ is valid in $Z Q$ if and only if $F$ is valid in $A$.

We can prove the following theorem quite similarly to Theorem 2.5.

Theorem 2.8. Any model of $\mathbf{D}$ can be embedded in some model of $\mathbf{D C}$.

Since any model of $\boldsymbol{D}$ has a submodel $Z$ which is a model of $D C$, we have the following corollary. 
Corollary 2.9. For any universal formula $F$ of $\mathscr{L}$ and any model $A$ of $D, F$ is valid in $Z$ if and only if $F$ is valid in $A$.

Any model of $(a, b) \cup\{0<1\}$, that is, any totally ordered abelian group with 1 contains $Z$ as a submodel. Hence, for any model $A$ of (a, b) $\cup\{0<1\}$, the set of open formulas valid in $A$ is included in the set of open formulas valid in $Z$ which equals to the set of open theorems of $\boldsymbol{D}$. The theory $\boldsymbol{D}$ is an open theory, that is, axiomatizable by only open formulas. Therefore, we have

TheOREM 2.10. The theory $D$ is the greatest element of the class of open consistent theories in $\mathscr{L}$ containing $(\mathrm{a}, \mathrm{b}) \cup\{0<1\}$.

\section{REFERENCES}

[1] Y. Komori, Super-Łukasiewics propositional logics, to appear.

[2] G. Kreisel and J. L. Krivine, Elements of Mathematical Logic, North-Holland, Amsterdam, 1967.

Shizuoka University 